\title{
Uncertain Robotic Manipulator System's Tracking Control Based on Fuzzy Adaptive Method
}

\author{
Huiqiu Du \\ School of Information and Electronic Engineering, Shandong Institute of Business \\ and Technology, Yantai, China \\ duhuiqiu@126.com
}

\begin{abstract}
The paper puts forward a control scheme by a computed torque controller plus a fuzzy compensator concerning the issue of tracking control of uncertain robotic manipulator. Computed torque controller is used for the nominal system and the fuzzy compensator is used to control the uncertain system. The parameters of fuzzy compensator are adaptively adjusted based on Lynapunov stability theory. The proposed scheme can guarantee the asymptotical stabilization of close-loop system. Simulation results are included to demonstrate the effectiveness and feasibility of our developed method.
\end{abstract}

Keywords: uncertainty; robotic manipulator; computed torque control; fuzzy adaptive control

\section{Introduction}

Computed torque method is an effective method in the tracking control of the robot, and the properties are very advantageous [1]. However, there exist two difficulties: 1) to complete the rapid computation for the robot's dynamic model; 2) to comprehend the dynamic model accurately in advance. They are caused by the poor robustness of computed torque method in the case of unknown model. In practice, it is difficult to get a relatively ideal robot dynamic model, besides, the parameters of the dynamic model may change in manipulation process and affected by various uncertain factors, such as environment and load variations. Whereas, this does not indicate that the computed torque method is invalid.

In recent years, many scholars present various tracking control schemes of uncertain robotic manipulator based on computed torque methods. Wijesoma [2] combined the variable structure control with the computed torque control to implement the tracking control, but the method involves the complicated inverse operation of inertia matrix; Neural network [3-4], as the functional approximation device, may emulate the concentration uncertainty of the system and superimpose on the computed torque controller. It can also realize the tracking of the desired trajectory. Moreover, the combination of many control algorithms like model reference adaptive control [5] algorithm and the computed torque method can also complete the tracking control of uncertain robotic manipulator.

Fuzzy control mainly depends on the operator's experience and is controlled by applying the fuzzy set theory which has the following features: it does not need to know the accurate mathematical model of the controlled object, and it just requires control experience or operating data. For some complex systems, such as robot system, electrohydraulic servo system, it is difficult to establish accurate mathematical model by the classical control theory, therefore it is more appropriate to use fuzzy control. Control rules are usually expressed by language variables, and can be realized by the simple software and hardware; It is easy to establish rules of language variables to implement real-time control; It has strong robustness and is not sensitive to changes of process parameters, 
especially, it is suitable for the time-varying nonlinear system; It can guarantee the system rapidly reach a steady state under the precondition of small overshoot or without overshoot, and shows the advantages of the nonlinear control. On the other hand, the fuzzy system can approximate any continuous function on the arbitrary precision, which also provides an important theory basis for the fuzzy system's application on identification.

This paper presents the strategy involving computed torque plus fuzzy control used to the tracking control of uncertain robotic manipulator. The basic idea is that the dynamic model of robot is divided into the known nominal system of structural parameters and the unknown uncertain system of structural parameters. For the nominal system, computed torque controller is used, and for the uncertain systems, the fuzzy logic system is used to approximate the centre of the uncertainty, the parameters of the fuzzy system will make adaptive adjusting based on Lynaponv stability theory. The closed-loop system is asymptotically stable in the controller [1-11].

\section{Preparations}

In recent years, many scholars have proved that the fuzzy system can approximate any continuous function at an arbitrary precision. The brief introduction will be given below.

Considering a multiple input and single output fuzzy system, its input variables: $x_{1}, x_{2}, \cdots, x_{n}$, are denoted by the vector: $x=\left[x_{1}, x_{2}, \cdots, x_{n}\right]^{T}$. The domains of the input variable $x$ and the output variable $y$ of the system are tightly set in the real space, i.e., $x, y \subset U \subset R^{n}$. The form of fuzzy rules is

$$
R^{j}: \text { if } x_{1} \text { is } A_{1}^{j} \text { and } x_{2} \text { is } A_{2}^{j} \text { and } \cdots \cdots \text { and } x_{n} \text { is } A_{n}^{j} \text { then } y \text { is } B^{j} \text {. }
$$

Here in $j=1,2, \cdots, M, M$ is rule number; $A_{i}{ }^{j}$ is the fuzzy set of $x_{i}$, belonging to the function $\mu_{A_{i}^{j}}\left(x_{i}\right), i=1,2, \cdots, n, B^{j}$ is the fuzzy set of $y$, belonging to the function $\mu_{B^{j}}(y)$. Assuming that the input variables belong to the Gauss function, i.e.,

$$
\mu_{A_{i}^{j}}\left(x_{i}\right)=\exp \left[-\frac{1}{2}\left(\frac{x_{i}-\bar{x}_{i}^{j}}{\sigma_{i}^{j}}\right)^{2}\right\rceil
$$

Membership function with output variables is fuzzy single point. Using the product inference method and weighted fuzzy algorithm, the output of the fuzzy system is

$$
y=f(x)=\frac{\sum_{j=1}^{M} \bar{y}^{j}\left(\prod_{i=1}^{n} \mu_{A_{i}^{j}}\left(x_{i}\right)\right)}{\sum_{j=1}^{M}\left(\prod_{i=1}^{n} \mu_{A_{i}^{j}}\left(x_{i}\right)\right)}
$$

If the parameters of input variables of the membership function $\bar{x}_{i}^{j}, \sigma_{i}^{j}$ have been set, only the parameters of the output variables of membership functions need to be adjusted. Then, the above expression can read as

$$
y=f(x)=\sum_{j=1}^{M} \bar{y}_{j} p_{j}(x)=\Theta^{T} \xi(x)
$$

where $\Theta=\left[\bar{y}_{1}, \bar{y}_{2}, \cdots, \bar{y}_{M}\right]^{T}, \xi(x)=\left[p_{1}(x), p_{2}(x), \cdots, p_{M}(x)\right]^{T}$, and

$$
p_{j}(x)=\frac{\prod_{i=1}^{n} \mu_{A_{i}^{j}}\left(x_{i}\right)}{\sum_{j=1}^{M}\left(\prod_{i=1}^{n} \mu_{A_{i}^{j}}\left(x_{i}\right)\right)}
$$


are called the fuzzy basis function. Eq. (4) is the fuzzy basis function expansion of the fuzzy system.

\section{Lemma (The Universal Approximation Theorem)}

Supposing $Y$ is the set of the fuzzy basis function expansion Eq. (3), the definition of FBF is given in Eq. (4). For any given continuous real function ${ }^{g}$ and any $\varepsilon>0$ in the close set $U \subset R^{n}$, there exists $f \in Y$ which leads to

$$
\sup _{x \in U}|g(x)-f(x)|<\varepsilon
$$

The universal approximation theorem provides the theory basis for the fuzzy system being used to almost all the nonlinear modeling problems. It shows that there exists fuzzy system with the form of Eq. (3) to approximate the given function at an arbitrary precision. The fuzzy logic system has the important function of using language information effectively with the uniqueness different from the other universal approximation devices (the polynomial approximation, the neural network approximation).

\section{Problem Description and the Controller Design}

For a serial robot with $n$ degrees of freedom, when the function of torque exists at each joint, we can use the Lagrange method to establish the robot dynamics equation as follows

$$
\begin{aligned}
& M(q) \ddot{q}+H(q, \dot{q})+\tau_{d}(q, \dot{q}, t)=\tau \\
& H(q, \dot{q})=C(q, \dot{q}) \dot{q}+G(q)+f(\dot{q})
\end{aligned}
$$

in the equation, $q, \dot{q}, \ddot{q}$ are respectively the position vector, velocity vector and acceleration vector of the $n \times 1$ dimension $(n \times 1 \mathrm{D})$ joints, $\tau$ is the corresponding force/Torque vector on the joint $n \times 1, M(q)$ is the Manipulator symmetric positive definite inertia matrix in $n \times n \mathrm{D}, C(q, \dot{q}) \dot{q}$ is the Centripetal force torque and Coriolis force in $n \times 1 \mathrm{D}, G(q)$ is the gravitational vector in $n \times 1 \mathrm{D}, f(q)$ is the frictional force vector and $\tau_{d}(q, \dot{q}, t)$ is the external disturbance in $n \times 1 \mathrm{D}$. When the dynamic model of robot system is precisely known and is not affected by external disturbances, namely, $M(q) C(q, \dot{q}) \dot{q}$ and $G(q)$ will also be precisely known, and $f(\dot{q})=\tau_{d}(q, \dot{q}, t)=0$. Insert the following computed torque controller

$$
\tau=M_{0}(q)\left(\ddot{q}_{d}-K_{v} \dot{e}-K_{p} e\right)+H_{0}(q, \dot{q})
$$

into Eq. (6), one has

$$
\ddot{e}+K_{v} \dot{e}+K_{p} e=0
$$

where $e=q-q_{d}$ is the trajectory tracking error in Eq. (7), and $q_{d}$ is the desired trajectory of the system, $K_{v}$ and $K_{p}$ the controller gain. Eq.(8) shows that the suitable choice of $K_{v}$ and $K_{p}$ may result in the tracking error asymptotically converged to zero. In the actual system, however, due to the uncertain elements impacting the robot, it is difficult to get the accurate dynamics model of robot system. Therefore Eq. (6) should be changed into

$$
\begin{gathered}
M(q)=M_{0}(q)-\Delta M(q) \\
C(q, \dot{q})=C_{0}(q, \dot{q})-\Delta C(q, \dot{q}) \\
G(q)=G_{0}(q)-\Delta G(q)
\end{gathered}
$$

here, $M_{0}(q), C_{0}(q, \dot{q}), G_{0}(q)$ are the known parts, and $\Delta M(q), \Delta C(q, \dot{q}) \dot{q}, \Delta G(q)$ describe the uncertainties of the system, insert Eq. (7) and Eq. (9) into (6), one has :

$$
\ddot{e}+K_{v} \dot{e}+K_{p} e=M_{0}^{-1}[\Delta M \ddot{q}+\Delta H]=\rho
$$


where $\rho=M_{0}^{-1}[\Delta M \ddot{q}+\Delta H]$ represents the concentration uncertainty of the system. The objective of this paper is: to design a computed torque controller and a fuzzy compensation controller and the two controllers can work together on the system (6), so that the robot system can track the given desired trajectory, i.e., to design the controller as:

$$
\tau=\tau_{0}+\tau_{c}
$$

where $\tau_{0}$ is the computed torque controller defined as in Eq. (7), and $\tau_{c}$ is the compensator controller designed below.

Using the concentration uncertainty $\rho(x)$ in the approximation robot system (6) in the fuzzy system mentioned in the above section, we suppose $\hat{\rho}(x)$ is the estimate value of the fuzzy approximation of $\rho(x)$, the compensation torque is selected according to the following equation:

$$
\tau_{c}=-M_{0}(q) \hat{\rho}(x)
$$

$\hat{\rho}(x)$ is defined as :

$$
\hat{\rho}(x \mid W)=W \xi
$$

where $w$ is a weight matrix, $\xi$ is fuzzy basis function vector, and $w^{*}$ is the optimal value of $w$ being a constant matrix and satisfying :

$$
W^{*}=\arg \min _{W \in \Omega_{f}}\left[\sup _{x \in U} \mid \hat{\rho}(x \mid W)-\rho(x)\right]
$$

Thus, the application of the concentration uncertainty of robot in the fuzzy system can be expressed as:

$$
\rho(x \mid W)=W^{*} \xi+\varepsilon
$$

where $\varepsilon$ is the approximation residual of the fuzzy system. Insert the overall control law Eq.(11) into the robot system Eq. (6), the closed-loop error equation of the system is:

$$
\ddot{e}+K_{v} \dot{e}+K_{p} e=\varepsilon+\tilde{W} \xi
$$

where $\tilde{W}=W^{*}-W$ is the weighting error matrix of fuzzy system.

For the convenience of analysis, we choose state variables $x=\left[e^{T}, \dot{e}^{T}\right]^{T}$, in which $e^{T}=\left[e_{1}, e_{2}\right]$. The closed-loop system can be written as state equation:

$$
\dot{x}=A x+B \rho
$$

where $A=\left[\begin{array}{cc}0 & I \\ -K_{p} & -K_{v}\end{array}\right], \quad B=\left[\begin{array}{l}0 \\ I\end{array}\right], \quad \rho=\varepsilon+\tilde{W} \xi$. And then we have the following theorem:

Theorem: apply the computed torque controller (7) and the fuzzy compensator controller (12) to the robot system (6), i.e., the selection control law (11) and take the following adaptive law:

$$
\dot{\tilde{W}}=-\Gamma^{-1} B^{T} P x \xi^{T}
$$

Then, the closed-loop system of the robot (6) is asymptotically stable. In the above equation $\Gamma=\operatorname{diag}\left(\Gamma_{1}, \Gamma_{2}, \cdots \Gamma_{n}\right), \Gamma_{i}>0(i=1,2, \cdots, n)$ is the gain matrix, $P$ is the symmetric positive definite solution of the following Racctia equation:

$$
A^{T} P+P A+P^{T} B B^{T} P+Q=0
$$

Proof: considering the Lyapunov function

$$
V=x^{T} P x+\operatorname{Tr}\left(\tilde{W}^{T} \Gamma \tilde{W}\right)
$$

For its following the closed loop system (17), we find the solution to the differential: 


$$
\begin{aligned}
\dot{V} & =x^{T}\left(A^{T} P+P A\right) x+\rho^{T} B^{T} P x+x^{T} P B \rho+2 \operatorname{Tr}\left(\tilde{W}^{T} \Gamma \dot{\tilde{W}}\right) \\
& =x^{T}\left(A^{T} P+P A\right) x+2 x^{T} P B \rho+2 \operatorname{Tr}\left(\dot{\tilde{W}}^{T} \Gamma \tilde{W}\right)
\end{aligned}
$$

Insert (17) and (18) into the above equation, one has:

$$
\dot{V}=-x^{T}\left(P^{T} B B{ }^{T} P+Q\right) x+2 x^{T} P B \varepsilon+2 x^{T} P B \tilde{W} \xi-2 T r\left(\xi x^{T} P B \tilde{W}\right)
$$

According to $x^{T} P B \tilde{W} \xi=\operatorname{Tr}\left(\xi x^{T} P B \tilde{W}\right)$, one obtains:

$\dot{V}=-x^{T}\left(P^{T} B B^{T} P+Q\right) x+2 x^{T} P B \varepsilon=-x^{T} Q x-\left(B^{T} P x-\varepsilon\right)^{T}\left(B^{T} P x-\varepsilon\right)+\varepsilon^{T} \varepsilon \leq-x^{T} Q x+\varepsilon^{T}$

Taking integration from two sides $t=0$ to $t=T$, we have:

$$
V(T)-V(0) \leq-\int_{0}^{T} x^{T} Q x d t+\int_{0}^{T} \varepsilon^{T} \varepsilon d t
$$

It is easy to $\operatorname{see} V(T)-V(0)<\infty$, which shows that $x$ and $W$ are bounded. The above equation can read as:

$$
\int_{0}^{T} x^{T} Q x d t \leq V(0)+\int_{0}^{T} \varepsilon^{T} \varepsilon d t
$$

Accordingly:

$$
\int_{0}^{T}\|\tilde{x}\|^{2} d t \leq k / \lambda_{\min }(Q)
$$

where $k=V(0)+\int_{0}^{T} \varepsilon^{T} \varepsilon d t, \lambda_{\min }(Q)$ is the minimum eigenvalue of matrix $Q$. So, for $x \in L_{2}$ according to Barblet lemma, we have:

$$
\lim _{t \rightarrow \infty} \tilde{x}(t)=0
$$

Thus, the closed-loop system is asymptotically stable.

\section{Simulation}

In this section, we will verify the algorithm proposed in this paper by an uncertainty second connecting rod manipulator system, let the dynamics equation of mechanical arm as Eq. (6), the parameter expressions are given as:

$$
\begin{gathered}
M(q)=\left\lfloor\begin{array}{cc}
\theta_{1}+\theta_{3}+2 \theta_{2} \cos q_{2} & \theta_{3}+\theta_{2} \cos q_{2} \\
\theta_{3}+\theta_{2} \cos q_{2} & \theta_{3}
\end{array}\right] \\
\mathrm{H}(\mathrm{q}, \dot{\mathrm{q}})=\left[\begin{array}{c}
-\theta_{2}\left(2 \dot{q}_{1} \dot{q}_{2}+\dot{q}_{2}^{2}\right) \sin q_{2} \\
\theta_{2} \dot{q}_{1}^{2} \sin q_{2}
\end{array}\right]
\end{gathered}
$$

where $\theta_{1}=I_{1}+\left(\frac{m_{1}}{4}+m_{2}\right) l_{1}^{2}, \theta_{2}=\frac{m_{2} l_{1} l_{2}}{2}, \theta_{3}=I_{2}+\frac{m_{2} l_{2}^{2}}{4}, m_{i}, l_{i}, I_{i}$ are respectively the quality of the $i$ th connecting rod, the length and the moment of inertia. We provide the following three simulations to illustrate the issues.

\subsection{Simulation 1}

Assuming that the model of the mechanical arm system is accurately known and is not affected by the uncertainty, the physical parameters are respectively $l_{10}=l_{20}=0.5 \mathrm{~m}, m_{10}=m_{20}=4 \mathrm{Kg}, I_{10}=I_{20}=0.2 \mathrm{kgm}^{2}$, and the tracking desired trajectory is $q d=[0.5 \cos t+0.2 \sin 3 t,-0.2 \sin 2 t-0.5 \cos t]^{\mathrm{T}}$. Using the computed torque controller (7) and choosing the control parameters $K_{v}=\operatorname{diag}(5.5,5.5), K_{p}=\operatorname{diag}(8,8)$, the tracking error gained by simulation is now 
shown in Fig. 1. It is thus clear that the desired trajectory can be tracked in a short time.
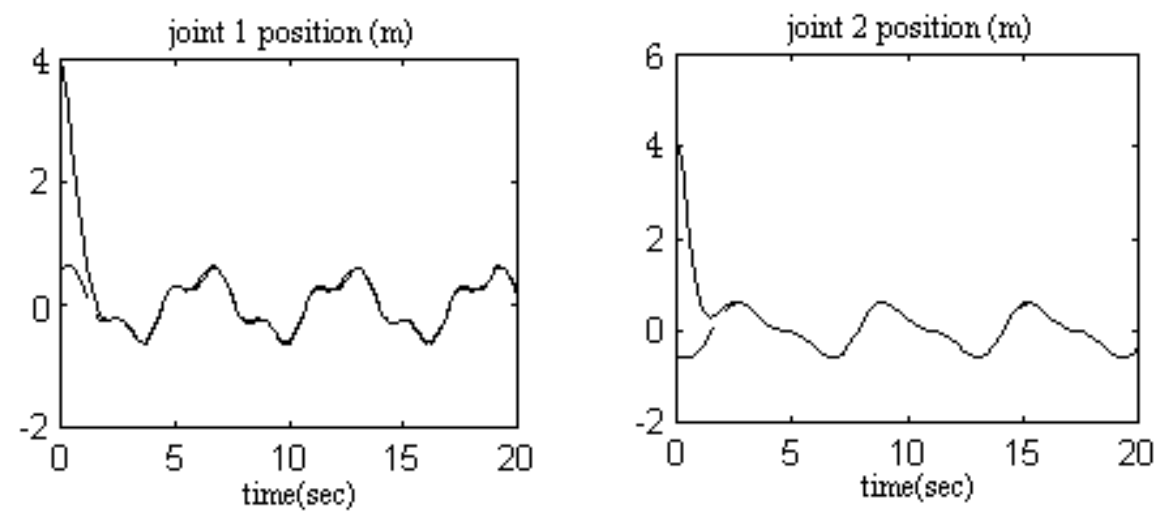

\section{Figure 1. Tracking Curve for Computed Torque Applied to the Precisely Known Robotic Manipulator System of Dynamic Control Model}

\subsection{Simulation 2}

Based on the above context, assuming that the physical parameters of the system are not precisely known, and the actual values of physical parameters are:

$$
l_{1}=l_{2}=0.5 \mathrm{~m}, m_{1}=m_{2}=8 \mathrm{Kg}, I_{1}=I_{2}=0.4 \mathrm{kgm}^{2}
$$

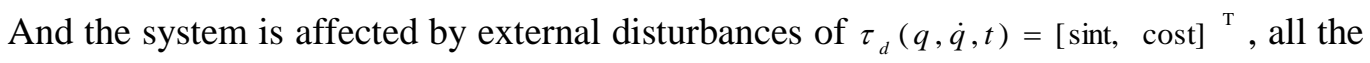
other parameters like the nominal value of physical parameters, desired trajectory, control parameters and simulation are totally the same. Then, the tracking curves gained are shown in Fig. 2 which demonstrates that the control performance of the computed torque control is very poor under the unknown parameters and the external disturbances.
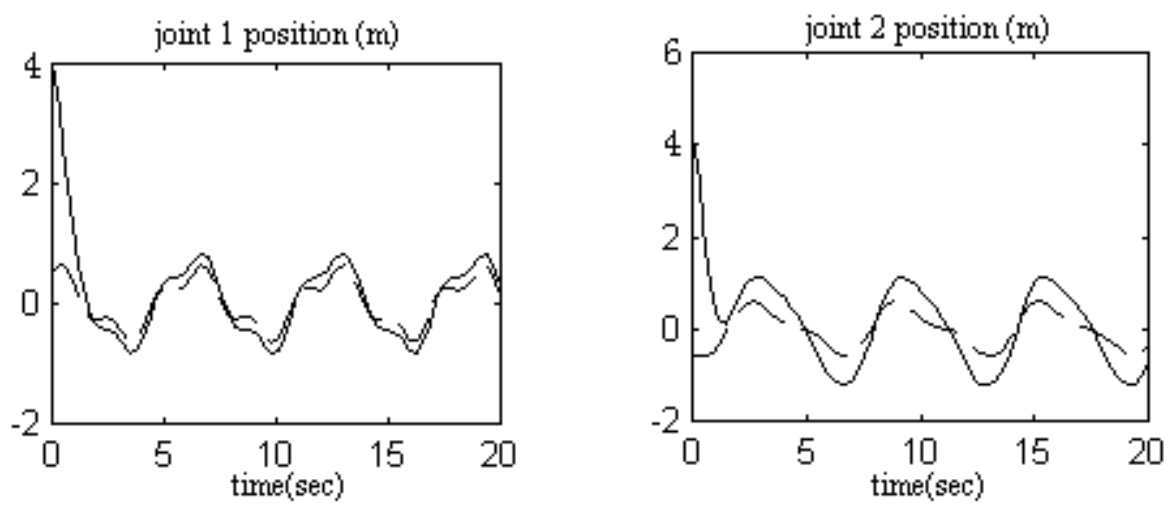

Figure 2. Tracking Curve for Computed Torque Applied to the Unknown Robotic Manipulator System of Dynamic Control Model

\subsection{Simulation 3}

Based on Simulation 2, we use the control strategy of theorem 1 to overlay fuzzy compensator controller on the computed torque controller (7), the membership function can be taken as:

$$
\mu_{F_{i}^{1}}\left(x_{i}\right)=\exp \left(-\left(x_{i}+1.5\right)^{2}\right) \quad \mu_{F_{i}{ }^{2}}\left(x_{i}\right)=\exp \left(-\left(x_{i}+0.5\right)^{2}\right)
$$




$$
\begin{gathered}
\mu_{F_{i}{ }^{3}}\left(x_{i}\right)=\exp \left(-\left(x_{i}{ }^{2}\right), \quad \mu_{F_{i}{ }^{4}}\left(x_{i}\right)=\exp \left(-\left(x_{i}-0.5\right)^{2}\right)\right. \\
\mu_{F_{i}{ }^{5}}\left(x_{i}\right)=\exp \left(-\left(x_{i}-1.5\right)^{2}\right)
\end{gathered}
$$

where, $x_{i}(i=1,2,3,4)$ represents the system state vector. The positive definite matrix $Q$ and the adaptive gain matrix $\Gamma$ are respectively expressed as $Q=\operatorname{diag}(10,10,10,10)$ and $\Gamma=\operatorname{diag}(7,7)$. The simulation results are shown in Fig. 3. It is obvious to see that the control strategies proposed in this paper can compensate for the influence of system uncertainties.
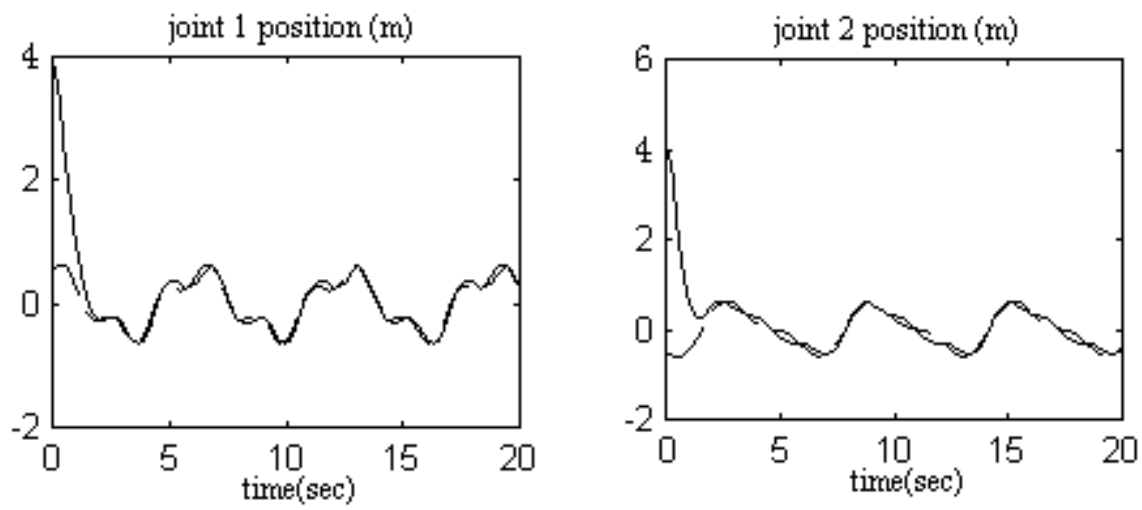

Figure 3. Tracking Curve for Computed Torque Plus Fuzzy Compensator Applied to the Unknown Robotic Manipulator of Dynamic Control Model

Note 1: Fig. 1, Fig. 2 and Fig. 3 presented above respectively indicate the motion trail of the two joints when the three control algorithms have effects on 2 degrees of freedom robotic manipulator (In the three figures, the sub-graph on the left refers to the motion track of Joint 1 , while the sub-graph on the right indicates the motion track of Joint 2.)

Note 2: In Fig. 1, Fig. 2 and Fig. 3 above, the horizontal axis represents time, the vertical axis indicates the position of the joint movement.

Note 3: In Fig. 1, Fig. 2 and Fig. 3 above, the dotted line represents the desired trajectory, the solid line indicates the actual track.

\section{Conclusion}

Computed torque algorithms are an effective method in controlling accurate robotic manipulator system of dynamic control model. But in practice, it is impossible to get accurate dynamic model of robotic manipulator. In order to solve this problem, we propose in this paper a compensator control strategy based on fuzzy adaptive controller, which is divided into two parts: the computed torque controller is used to control the known part of the system; the fuzzy logic system is used to study the uncertainty of robotic manipulator system so as to compensate for the uncertainty, its parameters implement adaptive adjustment. Under Lynapunov, the whole closed-loop system is asymptotically stable. The simulation results show that the algorithm is effective.

\section{Acknowledgements}

This work was financially supported by the Natural Science Foundation project of Shandong province of China(ZR2012FL11).

\section{References}


[1] J. J. Craig, "Introduction to Robotics: Mechanics and Control", Mass, Addison-Wesley, Massachusetts, (1986).

[2] S. W. Wijesoma, "Robust Trajectory Following of Robots Using Computed Torque Structures with VSS", Int. J. Control, vol.4, no.52, (1990).

[3] Z. Man, "A Robust Adaptive Sliding Mode Tracking Control Using a RBF Neutral Network for Robotic Manipulators", Proceedings of IEEE International Conference of Neural Network, (1995); San Diego, America.

[4] A. Ishiguro, "A Neural Network Compensator for Uncertainties of Robotics Manipulators", IEEE Transaction on Industrial Electronics, vol.6, no.39, (1992).

[5] S. K. Tso, "Variable Structure Model Reference Adaptive Control of Robot Manipulators", Proceedings of IEEE International Conference on Robotic and Automation, Sacramento, (1991); Sacramento, America.

[6] W. W. Chen, "Brief Communication: Uniform Ultimate Boundedness of a Fuzzy Logic Controlled in Industrial Robot”, J. of Robotic Systems, vol.9, no.18, (2001).

[7] J. J. Buckley, "Sugeno Type Controllers Are Universal Controllers", Fuzzy Sets and Systems, vol.53, (1993).

[8] H. Ying, "Sufficient Conditions on General Fuzzy Systems as Function Approximators", Automatica, vol.3, no.30, (1994).

[9] X. P. Shi and S. R. Liu, “A Survey of Trajectory Tracking Control for Robot Manipulators”, Control engineering of China, vol.1, no.18, (2001).

[10] S. Sheel and O. Gupta, "High Performance Fuzzy Adaptive PID Speed Control of a Converter Driven DC Motor", International Journal of Control and Automation, vol.1, no.5, (2012).

[11] L. P. Xi, Z. L. Chen and X. H. Qi, "Fast sliding mode variable structure control for manipulators with chattering suppression effect”, Electric Machines and Control, vol.7, no.16, (2012).

\section{Author}

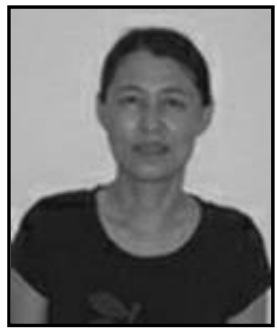

Huiqiu Du, she received her Master in engineering precision instruments and machinery (1985) from Harbin Normal University. Now he is Associate professor of School of Information and Electronic Engineering, Shandong Institute of Business and Technology. He makes a great deal research on robot technology and control. 